\title{
Effect of syntactic role and syntactic prominence on letter detection
}

\author{
JULIA E. MORAVCSIK and ALICE F. HEALY \\ University of Colorado, Boulder, Colorado
}

\begin{abstract}
Participants read passages and circled instances of a target letter. They made more correct detections on the when it was an adverb than when it was a definite article, when it occurred in a subject than in an object phrase, and when it was preposed than when it occurred normally. These results suggest that syntactic role affects letter detection even when meaning is controlled, and that giving greater syntactic prominence (i.e., salience or emphasis) affects letter detection even when both meaning and syntactic role are controlled. These findings pose a challenge to both the structural account of letter detection and the processing time account.
\end{abstract}

We have been using the letter detection task, in which participants read text and circle each instance of a target letter, to illuminate the cognitive processes employed in reading text (see Healy, 1994, for a summary). In previous studies, we showed that skilled readers miss more target letters when they are in very familiar words like the than when they are in less familiar words like thy. A unitization account (Drewnowski \& Healy, 1977; Healy, 1976) has been proposed to explain these findings, whereby participants in the letter detection task simultaneously process visual units at several levels, including letters and words. Identification of units at a higher level, such as the word level, may occur before complete identification of all of the units at a lower level, such as the letter level, especially when the units at the higher level are very familiar. Once units are identified at the higher level, the participants stop processing the component units at the lower levels and move on to process the next portion of text. By this account, the participants would be less likely to make letter detection errors on unfamiliar words because they would need to spend more time processing them at the word level before identification could occur. This extra time for word identification would provide the participants with more time for the simultaneous process of letter identification, which would naturally benefit letter detection. Thus, participants make fewer

This research was supported in part by Army Research Institute Contracts MDA903-93-K-0010 and DASW01-96-K-0010 to the University of Colorado (A.F.H., principal investigator). We are indebted to James Parker for help with the conduct and data tabulation of Experiments 1 and 3, to Barbara Schoephoerster for help with the design and conduct of Experiment 2, to Morton Ann Gernsbacher, Taimi Metzler, and Liang Tao for helpful discussions about linguistic issues, and to Keith Rayner, Seth Greenberg, and Kathryn Bock for helpful suggestions. Portions of this article concerning Experiment 2 were reported at the Rocky Mountain Psychological Association Meeting, Las Vegas, April 1994. Correspondence concerning this article should be addressed to A. F. Healy, Department of Psychology, Campus Box 345 , University of Colorado, Boulder, CO 80309-0345 (e-mail: ahealy@ clipr.colorado.edu). letter detection errors on unfamiliar words because identification of word units occurs less rapidly.

Support for this unitization account has been provided by a number of previous studies that manipulated perceptual factors. For example, alternating capital and small letters (Drewnowski \& Healy, 1977) or using an unfamiliar font (Schneider \& Healy, 1993) results in a decrease in letter detection errors in common words like the, presumably because these manipulations distort the familiar visual configuration of the common words.

In a recent study (Moravcsik \& Healy, 1995), we showed that word meaning, not just perceptual factors, influences letter detection. We proposed that word identification would not be complete until the reader accessed the meaning of the word. Under this assumption, familiarity of meaning as well as familiarity of visual configuration should affect the time to identify a word and, hence, the time devoted to the identification of the individual letters that constitute the word. More generally, we proposed a processing time explanation of letter detection, according to which the time spent processing a word is the crucial determinant of letter detection errors in that word, and participants are less likely to make a detection error on a letter in a word whenever they spend more time processing that word, for any reason.

An alternative account of letter detection has been proposed by Koriat and Greenberg (1994), who have emphasized structural syntactic factors. According to their structural account, letters in function words like the are often not detected because function words play an important role in the initial processing of a sentence, establishing the sentence's structural frame, but then they recede into the background during subsequent sentence processing. The greater proportion of letter detection errors on function words than on content words is then explained with the assumption that the detection task reflects processing during this subsequent stage.

In the present study, we first attempted to provide a stronger test of the importance to letter detection of the specific syntactic role of a word in the text. Both our pro- 
cessing time version of the unitization account of letter detection and the structural account would predict that the specific syntactic role of the test word would affect the proportion of letter detection errors on that word. We then attempted to explore effects of syntactic structure that have not been previously addressed by either the unitization account or the structural account.

\section{EXPERIMENT 1}

In our previous study (Moravcsik \& Healy, 1995), we found that participants made fewer letter detection errors on the word the when it was used with an unfamiliar meaning and syntactic role. Specifically, in two experiments, we compared the standard use of the word the as a definite article either to a new, artificial use as a content word referring to a Thai spice or to an existing, natural, but unusual use involving the citation form, in which the word the was used as a content word referring to the definite article itself. In the present experiment, we compared two different uses of the word the that differ in frequency but that are not artificial or unusual. Both uses are listed in a standard dictionary (see, e.g., Random House, Inc., 1987, p. 1966). The more frequent use was the standard one as a definite article and the less frequent use was as an adverb that modifies an adjective or adverb in the comparative degree (e.g., none the wiser). Both the processing time version of the unitization account (Moravcsik \& Healy, 1995) and the structural account (Koriat \& Greenberg, 1994) of letter detection lead to the prediction of a greater proportion of letter detection errors on the more frequent than on the less frequent use of the word the.

\begin{abstract}
Method
Participants. Twenty-four undergraduate students at the University of Colorado, Boulder, served as participants for credit in a course on introductory psychology. In this and the following experiments, all participants indicated that they were native speakers of English.

Materials. A 172-word prose passage in the form of a letter from a mother to her daughter was created. The passage contained 106 instances of the target letter $e$. There were 12 instances of the word the altogether, with 6 of them as a definite article in a noun phrase and 6 in an adverbial phrase, which we will refer to as an "adverb" use, following a standard dictionary (Random House, Inc., 1987, p. 1966). In the adverb use, the word the modifies an adjective or adverb in the comparative degree. See Table $l$ for sample sentences.

In this and the following experiments, no instance of the word the occurred as the first word of a sentence or the first or last word of a line, and two four-alternative multiple-choice comprehension questions followed the passage. The primary purpose of the comprehension test was to motivate the participants to read the passages for meaning.

Procedure. The participants were tested individually. The participants were instructed to read the passage "as quickly as you can (while still reading for comprehension), but whenever you come to an $e$ please circle it .... If at any time you realize that you have missed a word with the letter $e$ in it, do not go back to that word." The participants were told that at the end they would be given a few questions to test their comprehension. Although they were told to read quickly, their reading was not timed.
\end{abstract}

\section{Results}

Comprehension performance was adequate; the overall proportion of correct responses was .750 .
Table 1

Sample Sentences From Experiments 1, 2, and 3 (With Critical Test Words in Bold)

\section{Experiment 1}

Definite article: As you start your first year away from the family in college, I hope you remember ....

Adverb: Third, get lots of sleep and you will feel the better for it.

\section{Experiment 2}

Passive: Thus the spoon can be given to Baby by the parents.

Active: Thus the parents can give the spoon to Baby.

Experiment 3

Normal object: My policy is I do the pots and pans right away .... Preposed object: My policy is the pots and pans I do right away .... Normal subject: I was really embarrassed yesterday because the landlord came in ...

Preposed subject: I was really embarrassed yesterday because the landlord, he came in .

Participants made a significantly smaller proportion of letter detection errors on the word the when it occurred in its less frequent use as an adverb (.319) than when it occurred in its more frequent use as a definite article (.486), by an analysis with participants as the random effect $\left[F(1,23)=19.69, M S_{\mathrm{e}}=.0169, p<.001\right]$ and by an analysis with items as the random effect $[F(1,10)=7.66$, $\left.M S_{\mathrm{e}}=.0109, p=.019\right]$. The results support the hypothesis that the syntactic role of the word the affects letter detection on that word, even when neither role being considered is artificial or unusual. These findings are consistent with both our processing time version of the unitization account and the structural account of letter detection.

\section{EXPERIMENT 2}

In Experiment 1, we found a large effect of syntactic role on letter detection. However, in this effect, syntactic role was confounded with meaning. In Experiment 2, we examined an effect of the syntactic role of the noun phrase that contained the word the, when both the meaning and the syntactic role of the test word the were held constant (it was used as a definite article in each case) and the semantic role of the noun phrase and sentence meaning were also controlled.

Specifically, in Experiment 2, participants performed the letter detection task on one of two versions of a prose passage that differed in terms of whether a given sentence was in the active or passive voice. This manipulation ensured that a given noun phrase that included the familiar test word the would be a subject in one version and an object (either a direct object or an object of the preposition $b y$ ) in the other version. On the basis of previous findings (Moravcsik \& Healy, 1995, Experiments 1 and 4), we expected that participants would make fewer detection errors on the word the when it occurred in a subject noun phrase than when it occurred in an object noun phrase. This difference between subject and object might be greater for passive sentences; it has been 
argued (see, e.g., Ferreira, 1994; Givon, 1992) that speakers and writers are most likely to choose the passive form of a sentence (rather than the unmarked active form) when they want to place the subject noun phrase in a position of prominence or emphasize it.

\section{Method \\ Participants. Twenty-eight undergraduate students at the Univer- sity of Colorado, Boulder, served as participants for credit in a course on introductory psychology. \\ Materials. Two versions of a prose passage were created, loosely based on an excerpt from a book on infant care, with 119 words in one version and 121 words in the other version. Each version contained 30 instances of the target letter $h$. There were 14 instances of the word the in each version, with 7 of these instances occurring in a subject noun phrase and the remaining 7 occurring in an object noun phrase. The two passage versions differed in terms of whether a given sentence was in the active or passive voice, so that a given noun phrase that included the word the occurred as a subject in one passage version and as an ob- ject in the other passage version. See Table 1 for sample sentences. \\ Procedure. The participants were tested in small groups. Each par- ticipant was given one version of the prose passage. The assignment of participants to passage version was made on the basis of time of arrival for testing and seat assignment according to a fixed rotation, so that an equal number of participants received each passage version. The in- structions were like those used in Experiment 1 except that the target letter was changed to $h$. (We changed the target simply to increase the generalizability of the findings.)}

\section{Results}

Comprehension performance was adequate; the overall proportion of correct responses was .679 .

As shown in Table 2, participants made a significantly smaller proportion of letter detection errors on the word the when it occurred in a subject noun phrase than when it occurred in an object noun phrase, by an analysis with participants as the random effect $\left[F(1,27)=8.70, M S_{\mathrm{e}}=\right.$ $.0257, p=.007]$ and by an analysis with items as the random effect $\left[F(1,24)=4.39, M S_{\mathrm{e}}=.0106, p=.044\right]$. Although the advantage for subject noun phrases appears somewhat larger for passive than for active sentences, there was no effect of voice and no interaction of voice and syntactic role $(F<1$ in each case by participants and by items).

These findings are predicted by neither Koriat and Greenberg's (1994) structural account of letter detection nor our processing time version of the unitization account (Moravcsik \& Healy, 1995). They may be consistent with the processing time hypothesis, but only if it is the case that readers spend more time processing subject noun phrases than object noun phrases. This is a controversial issue. It has been observed that speakers and writers typically put the topic of a sentence, by which we mean "the entity ... about which something is said" (Crystal, 1991, p. 354), in the subject position (see, e.g., Givon, 1992). But it has also been observed that speakers and writers typically put given information (presuppositions) before new information (assertions) (see Costermans \& Hupet, 1977). These two observations lead to contradictory positions concerning the relative time spent processing noun phrases in the subject and object positions. On the one hand, it seems likely that readers would spend more processing time on noun phrases that constitute the topic of the sentence than they would spend on other noun phrases, which would imply that participants would spend more time processing noun phrases in the subject position than in the object positions. On the other hand, readers, like speakers, may spend more processing time on noun phrases that consist of new information than on those that consist of given information (see Bock, 1982, p. 37, for a relevant discussion of speech production), which would imply that participants would spend more time processing noun phrases in the object positions than in the subject position. Studies of eye movements during reading may provide observations directly relevant to this issue; however, an earlier study by Rayner (1977) revealed that readers fixated longer on verbs than on either subjects or objects, which did not differ from each other.

\section{EXPERIMENT 3}

We wondered whether the advantage we found for words in a subject noun phrase over an object noun phrase could be attributed to the fact that the words in a subject noun phrase occurred earlier in the sentence, although we had written the passage so that no instance of the word the occurred as the first word in a sentence. Gernsbacher (1990) reviewed evidence for an advantage in recall of the participant mentioned first in a sentence including two participants. Finding an effect of word position in a sentence would pose a challenge for both the structural account of letter detection and the processing time version of the unitization account. However, it is possible that word position in the sentence affects processing time as well as recall, and by the processing time explanation, letter detection errors are determined primarily by the time devoted to processing the words containing the target letter.

To vary position within the sentence while holding constant the syntactic role and meaning of the noun phrase as well as the syntactic role and meaning of the test word the, we used a prepose or left-dislocation manipulation on object noun phrases in Experiment 3. This manipulation, like the passive manipulation, has the effect of making the manipulated noun phrase function as the sentence topic and, thus, placing it in a position of prominence (i.e., salience or emphasis) within the sentence (see, e.g., McKoon, Ratcliff, Ward, \& Sproat, 1993, for a discussion of syntactic prominence). We also used a different preposing manipulation for subject noun phrases. This subject manipulation did not change the location of the noun phrase within the sentence (because the location was already in the left part of the sentence). Instead it involved adding a comma followed by a pronoun after the noun phrase for emphasis. Like the object manipulation, the subject manipulation lent greater prominence to the noun phrase and thereby encouraged the readers to treat it as the sentence topic. 


\section{Method}

Participants. Forty-eight undergraduate students at the University of Colorado, Boulder, served as participants. Thirty of them were in a course on cognitive psychology and participated as part of a class demonstration on their 1st day of class before any other material concerning cognitive psychology was presented. The remaining 18 participated for credit in a course on introductory psychology. All participants indicated that they had not previously participated in a letter detection experiment.

Materials. Two versions of a 249 -word prose passage were created in the form of a dialogue between two roommates. One version contained 55 instances of the target letter $h$, and the other version contained 56 instances. There were 27 instances of the word the in each version, including 10 critical instances. Eight of the critical instances occurred in object noun phrases; in each version, four of these critical instances occurred in the normal location after the verb phrase and four were preposed so that they occurred earlier in the sentence. Across the two passage versions, each critical instance was used in both the normal and preposed form. The remaining two of the critical instances occurred in subject noun phrases. In each version, one of these critical in stances occurred in its normal form and the other occurred in a preposed form with an added comma and pronoun after it for emphasis, instead of occurring at an earlier position in the sentence. Again, across the two versions, each of these critical instances was used in both forms. See Table 1 for sample sentences.

Procedure. The cognitive psychology students were tested in a group in their classroom. The introductory psychology students were tested individually. The instructions and procedure were like those used in Experiments 1 and 2; the target letter was $h$.

\section{Results}

Comprehension performance was adequate; the overall proportion of correct responses was .812 .

The subject and object phrases occurred in different sentences in Experiment 3, unlike in Experiment 2. Further, the preposing manipulation changed the order of the words in the object phrases but added a word in the subject phrases. Hence, any overall difference between the subject and object phrases would be difficult to interpret in the present experiment. However, it would be of interest to determine whether any preposing effect was of similar magnitude in the subject and object phrases. In fact, as shown in Table 2, it was found that participants made a significantly smaller proportion of letter detection errors (i.e., misses) on critical instances of the word the when they occurred in the preposed form than when they occurred in the normal form, by an analysis with participants as the random effect $\left[F(1,47)=5.02, M S_{\mathrm{e}}=\right.$ $.0937, p=.028]$. This effect of preposed status was found for both subject and object phrases; neither the main effect of grammatical role nor the interaction of grammat-

Table 2

Proportion of Detection Errors on the Word the in Experiments 2 and 3 as a Function of Syntactic Role and Voice or Preposed Status

\begin{tabular}{ccc}
\hline & \multicolumn{2}{c}{ Syntactic Role } \\
\cline { 2 - 3 } Experiment & Subject & Object \\
\hline Experiment 2 & & \\
Active & .542 & .625 \\
Passive & .545 & .640 \\
Experiment 3 & & \\
Preposed & .521 & .484 \\
Normal & .583 & .620 \\
\hline
\end{tabular}

ical role and preposed status was significant, by an analysis with participants as the random effect $[F(1,47)<$ 1.07 in each case]. We did not conduct an analysis with items as the random effect that included the factor of subject and object phrases because there were only two subject phrases. Instead, we examined only the effect of preposed status using items as the random effect (and including all 10 items) and found the effect to be significant with a one-tailed test $[t(9)=1.87, p=.046]$.

These results indicated that preposing a phrase decreased the proportion of letter detection errors in that phrase, whether the preposing was achieved by moving the phrase to a position of greater prominence in the sentence or by adding a comma and a pronoun after the noun phrase to give the phrase greater prominence. Hence, Experiment 3 indicated that position within the sentence did influence letter detection errors. These findings pose a challenge to both the structural account of letter detection and the processing time version of the unitization account.

There were no differences between subject and object phrases in the present experiment. However, the subject and object phrases occurred in different sentences, so that semantic role was not controlled and, hence, the subject and object phrases were not comparable, as they were in Experiment 2. Therefore, the lack of an effect of syntactic role in the present experiment does not undermine the findings in Experiment 2 that syntactic role did affect letter detection when semantic role was controlled.

\section{DISCUSSION}

In summary, in Experiment 1, we found that fewer letter detection errors were made on the word the when it occurred as an adverb than as a definite article. This finding is consistent with both Koriat and Greenberg's (1994) structural account of letter detection and our processing time version of the unitization account (Moravcsik \& Healy, 1995). However, in Experiment 2, we found that noun phrase syntactic role affected letter detection when the meaning and syntactic role of the test word the and the semantic role of the noun phrase and sentence meaning were controlled. Specifically, we found fewer letter detection errors on the word the when it occurred in the subject of a sentence than when it occurred in an object. Further, in Experiment 3, we found that letter detection errors in a given noun phrase were decreased by a preposing manipulation that either moved the noun phrase to a position of greater prominence in the sentence or added a comma and pronoun after the noun phrase to give it greater prominence. This finding occurred even though the syntactic and semantic roles of the noun phrases, the meaning of the sentence, and the meaning and syntactic role of the test word the were all held constant. These results from Experiments 2 and 3 pose a problem for both accounts of letter detection.

At first glance, the effects of syntactic role and syntactic prominence on letter detection may seem to support Koriat and Greenberg's (1994) structural account, according to which syntactic function affects detection errors. However, the structural account does not differentiate between subject and object roles or between normal noun phrases and those that have been preposed, but instead focuses only on the difference between function and content words. Hence, the structural account would need to be expanded or modified in order to account for the findings in Experiments 2 and 3. Likewise, our processing time explanation of letter detection (Moravcsik \& Healy. 1995) would need to be expanded or modified to account for the findings. Specifically, the processing time explanation would be consistent with the present pattern of results only given three assumptions. First, it 
must be assumed that readers devote more processing time to the subject of a sentence than to other noun phrases in the sentence. Second it must be assumed that readers devote more processing time to preposed phrases than to the same phrases occurring normally within the sentence. Third, because the advantage for preposing occurred even when the preposing was achieved by adding a comma and a word after the test word the, it must be assumed that the relevant processing time is not limited to that involving initial word identification; rather, it must include both the initial fixation duration on the test word and the time spent during any regressive eye movements to the test word. These three assumptions require further empirical verification. In addition future research should be directed at exploring additional syntactic factors with the goal of gaining a fuller understanding of the effects of syntactic role and prominence on letter detection. Nevertheless, the present study makes it clear that syntactic role and prominence do have important effects on letter detection, even when semantic role and sentence meaning are controlled.

\section{REFERENCES}

Bock, J. K. (1982). Toward a cognitive psychology of syntax: Information processing contributions to sentence formulation. Psychological Review, 89, 1-47.

Costermans, J., \& HuPET, M. (1977). The other side of JohnsonLaird's interpretation of the passive voice. British Journal of Psychology, 68, 107-111

CRYSTAL, D. (1991). A dictionary of linguistics and phonetics (3rd ed.) Oxford: Basil Blackwell.

Drewnowski, A., \& Healy, A. F. (1977). Detection errors on the and and: Evidence for reading units larger than the word. Memory \& Cognition, 5, 636-647
FERREIRA, F. (1994). Choice of passive voice is affected by verb type and animacy. Journal of Memory \& Language, 33, 715-736.

GERNSBACHER, M. A. (1990). Language comprehension as structure building. Hillsdale, NJ: Erlbaum.

Givon, T. (1992). The grammar of referential coherence as mental processing instructions. Linguistics, 30, 5-55.

HEALY, A. F. (1976). Detection errors on the word the: Evidence for reading units larger than letters. Journal of Experimental Psychology: Human Perception \& Performance, 2, 235-242.

Healy, A. F. (1994). Letter detection: A window to unitization and other cognitive processes in reading text. Psychonomic Bulletin \& Review, 1, 333-344.

Koriat, A., \& Greenberg, S. N. (1994). The extraction of phrase structure during reading: Evidence from letter detection errors. Psychonomic Bulletin \& Review, 1, 345-356.

McKoon, G., Ratcliff, R., Ward, G., \& Sproat, R. (1993). Syntactic prominence effects on discourse processes. Journal of Memory \& Language, 32, 593-607.

Moravcsik, J. E., \& Healy, A. F. (1995). Effect of meaning on letter detection. Journal of Experimental Psychology: Learning, Memory, \& Cognition, 21, 82-95.

RaNDOM HousE, INC. (1987). The Random House dictionary of the English language (2nd ed., unabridged). New York: Author.

RAYNER, K. (1977). Visual attention in reading: Eye movements reflect cognitive processes. Memory \& Cognition, 5, 443-448.

Schneider, V. I., \& HeAly, A. F. (1993). Detecting phonemes and letters in text: Interactions between different types and levels of processes. Memory \& Cognition, 21, 739-751.

(Manuscript received December 24, 1996; revision accepted for publication July $17,1997$. ) 